\title{
Power-Amplifier Transient Tracking in OFDM
}

\author{
David Bateman, Sebastien Simoens, Marc de Courville and Gilbert Julien \\ Motorola Labs - Paris, Espace Technologique; Saint Aubin, 91193 Gif-Sur-Yvette, FRANCE \\ * Motorola WBSG, 207 Route de Ferney, 1218 Grand Saconnex, Geneva, SWITZERLAND
}

\begin{abstract}
The effect of the transmitter gain transient when the power-amplifier is switched on and off (to conserve battery power) is investigated for OFDM systems. A simple receiver-side correction technique is proposed for the HIPERLAN/2 OFDM system, and the improvement in performance is demonstrated.
\end{abstract}

\section{INTRODUCTION}

By its nature, any wireless communication system is likely to be battery powered. Therefore it is important to consider means of conserving the batteries power. The most obvious technique is to turn off parts of the system when they are not in use. If we turn off only the most power hungry of the components, the gain in terms of battery life can be similar, but the system can be simplified. For instance, the RF component that consumes the most power is the power-amplifier in the transmitter. By only switching this component on when transmitting, the power consumption can be greatly reduced.

However, when an amplifier is switched on it has a settling time before its gain is stable. Understanding the effects of this gain transient on the system performance is critical when evaluating performance. OFDM systems such as IEEE 802.11a and HIPERLAN/2 (HL/2), which have almost the same physical layer, are particularly sensitive to such non-linear gain. This paper, studies the effect of a power-amplifier gain transient with a commercially available amplifier; shows that the HL/2 standard can be met in the presence of this transient, measures the performance degradation due to this gain transient, and suggests a simple correction scheme.

\section{TYPICAL POWER-AMPLIFIER TRANSIENT RESPONSE}

We chose to measure the transient behavior of the commercially available ARAFTEK 7501 power-amplifier module. This GaAs amplifier is suitable for $5 \mathrm{GHz}$ applications and has a $1 \mathrm{~dB}$ compression point of $23 \mathrm{dBm}$. To simulate a typical mobile application within the context of a $\mathrm{HL} / 2$ system the ARAFTEK amplifier was switched on and off with a cycle of $2 \mathrm{~ms}$, to simulate the HL/2 frame-rate. The power-amplifier was switched on for only $0.4 \mathrm{~ms}$ of that time. As we wished to see a worst-case situation, we drove the amplifier with a $7 \mathrm{dBm} \mathrm{CW}$ input, which gave a compressed output signal.

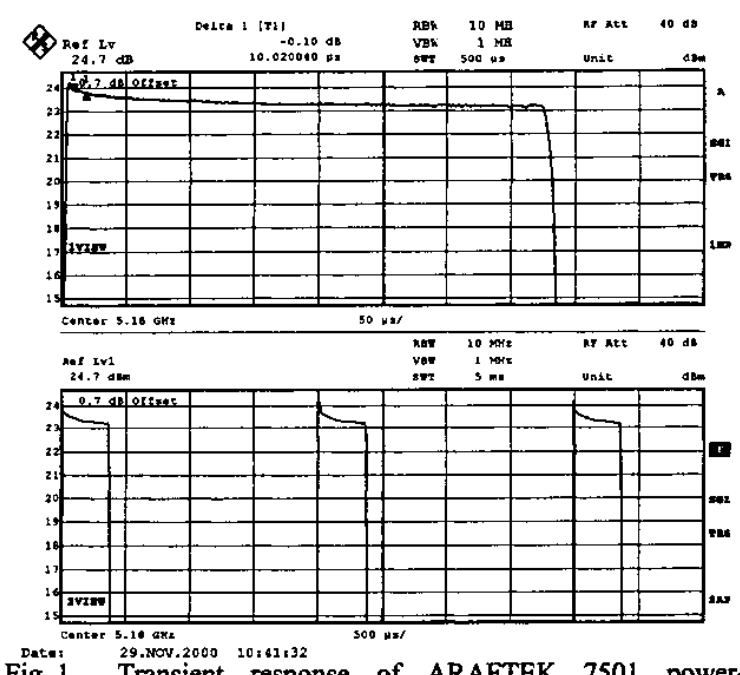

Fig. 1. Transient response of ARAFTEK 7501 poweramplifier with cycle of $0.4 \mathrm{~ms}$ On, $1.6 \mathrm{~ms}$ OFF.

Fig 1 demonstrates the transient behavior of this amplifier. Two major effects can be seen: an overshoot as the amplifier is turned on, and a gradual reduction in the gain of the amplifier due to thermal effects. The HL/2 standard, allows $6 \mu$ s switching time between receive and transmit modes, and so only the thermal effect needs to be considered when modeling the effect of the poweramplifier transient.

\section{EFFECT OF POWER-AMPLIFIER TRANSIENT ON PERFORMANCE}

The gain transient of the power-amplifier is in fact a non-linearity that causes inter-modulation of the OFDM carriers, introducing an additionally noise effect. Additionally, after passing through the channel, this nonlinearity affects the cyclic nature of the OFDM coding. 
The power-amplifier adds a gain that varies with the time index of the transmitted blocks. This destroys the diagonal property of the transmission channel when inserting the cyclic prefix, thus creating inter-carrier interference (ICI).

When adopting a digital modeling of the gain transient, its effects can be described by the following equations:

$$
x(n T)=\left(1+a b^{n}\right) s(n T)
$$

where $x(t)$ is the time domain signal sent through the channel. Matching the above parameters $a$ and $b$ to the curves in Fig 1 we find that $a=0.0839$ and $b=0.9997$

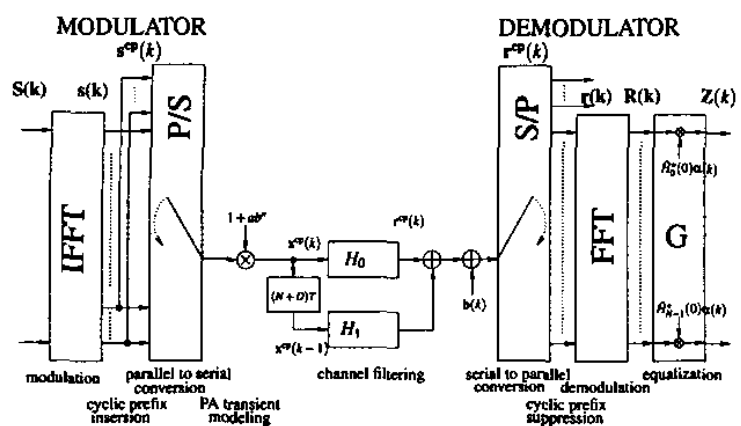

Fig. 2. Digital model of the OFDM system with poweramplifier transient effect

To complete the notation, the classical operations for OFDM [1] modulation of a vector $\mathbf{S}(k)$ ( $k$ being the block index) generating the time domain samples $\mathbf{s}(k)$ and cyclic prefix insertion transforming $s(k)$ into $s^{c p}(k)$ are shown below and depicted in Fig 2:

$$
\begin{gathered}
s_{n}(k)=s((k N+n) T), 0 \leq n<N \\
\mathbf{S}(k)=\left(S_{0}(k), \cdots, S_{N-1}(k)\right)^{t} \\
\mathbf{s}^{c p}(k)=\left(s_{N-D}(k), s_{N-D+1}(k), \cdots, s_{N-1}(k), s_{0}(k), s_{1}(k), \cdots, s_{N-1}(k)\right)^{y} \\
\mathbf{s}(k)=\left(s_{0}(k), \cdots, s_{N-1}(k)\right)^{t}=F_{N}^{-1} \mathbf{S}(k)
\end{gathered}
$$

where $N$ denotes the FFT size, $D$ the duration of the cyclic prefix, in number of samples, and $T$ the sampling rate. For $\mathrm{HL} / 21 / T=20 \mathrm{MHz}, N=64$ and $D=16$.

After some calculus, it can be shown, after guard interval stripping, that the matrix in equation (3) models the filtering by the transmission channel $h=\left(h_{0}, \cdots, h_{t-1}, 0, \cdots, 0\right)^{\gamma}$ with $l \leq D$. All the components of the matrices are blocks of size $D \times D$ :

$$
\begin{aligned}
& H_{0}=\left[\begin{array}{ccccc}
h_{0} & 0 & \cdots & \cdots & 0 \\
\vdots & \ddots & \ddots & & \vdots \\
h_{l-1} & & \ddots & \ddots & \vdots \\
\vdots & \ddots & & \ddots & 0 \\
0 & \cdots & h_{t-1} & \cdots & h_{0}
\end{array}\right] \\
& H_{1}=\left[\begin{array}{ccccc}
0 & \cdots & h_{l-1} & \cdots & h_{1} \\
\vdots & \ddots & & \ddots & \vdots \\
\vdots & & \ddots & & h_{t-1} \\
\vdots & & & \ddots & \vdots \\
0 & \cdots & \cdots & \cdots & 0
\end{array}\right] \\
& B=\operatorname{diag}\left(b^{0}, b, \ldots, b^{D-1}\right)
\end{aligned}
$$

and $\beta=b^{N / 4}$. The received time domain vector $\mathbf{r}(k)$ to be demodulated is thus expressed as (noiseless case):

$$
\mathbf{r}(k)=\left(H_{\text {circ }}+H_{\text {trans }}(k)\right) \mathrm{s}(k)
$$

Note that $H_{\text {circ }}$ is circular and corresponds to a circular convolution [2] by the channel (yielding no ICI) whereas $H_{\text {trans }}(k)$ includes the effect of the power-amplifier transient Finally, the received symbols at the FFT output are:

$$
R(k)=\operatorname{dia} g\left(F_{N} h\right) S(k)+a b^{k(N+D)} F_{N} H_{\text {tran }\{k)} F_{N}^{-1}
$$

For typical values of $a$ and $b$, such as those provided before, it can be observed that $B \approx I_{D}$ (identity matrix of size $\mathrm{D}$ ) and $\beta \approx 1$. Thus, equation (6) is approximated by:

$$
R(k) \approx\left(1+a b^{k(N+D)}\right) \operatorname{diag}\left(F_{N} h\right) S(k)
$$

According to equation (7), the effect of the poweramplifier transient can be viewed as a simple scalar gain $g(k)=1+a b^{k(N+D)}$ affecting the channel, and varying along the frame or burst (as $k$ increases). In $\mathrm{HL} / 2$ and $802.11 \mathrm{a}$, the channel is estimated in the preamble of the burst. Thus, neglecting the variation of $g(k)$ leads to an error in Viterbi metrics computation [1] which degrades the performance. Another source of degradation comes from the approximation in equation (7), which assumes that $H_{\text {trans }}(k)$ is circular thus neglecting the ICI. This second effect is produced by the variation of the gain inside the

$$
\left[\begin{array}{cccc}
H_{0} & 0 & 0 & H_{1} \\
H_{1} & H_{0} & 0 & 0 \\
0 & H_{1} & H_{0} & 0 \\
0 & 0 & H_{1} & H_{0}
\end{array}\right]+a b^{k(N+D)}\left[\begin{array}{cccc}
\beta H_{0} B & 0 & 0 & H_{1} B \\
\beta H_{1} B & \beta^{2} H_{0} B & 0 & 0 \\
0 & \beta^{2} H_{1} B & \beta^{3} H_{0} B & 0 \\
0 & 0 & \beta^{3} H_{1} B & \beta^{4} H_{0} B
\end{array}\right]=H_{\text {circ }}+H_{\text {trans }}(k)
$$


OFDM symbol, whereas the first effect is due to the gain variation from one OFDM symbol to the next. The first can be easily compensated if $g(k)$ is known or estimated, whereas the ICI will be much harder to compensate.

The average Euclidean norm of $H_{\text {trans }}(k)$ over the realizations of the channel corresponds to the Error Vector Magnitude (EVM) that would be measured at the receiver when no compensation method is applied. It is plotted as a function of the OFDM symbol in Fig 3. The limiting value of the error (about $-24 \mathrm{~dB}$ ) is sufficient to cause degradation in the bit error rate performance of the higher order modulations, such as QAM64. We also plotted on Fig. 3 the error due to ICI only, assuming $g(k)$ is perfectly known, and it can be observed that this error is negligible. Therefore, in the following we introduce a compensation scheme based on the estimation of $g(k)$.

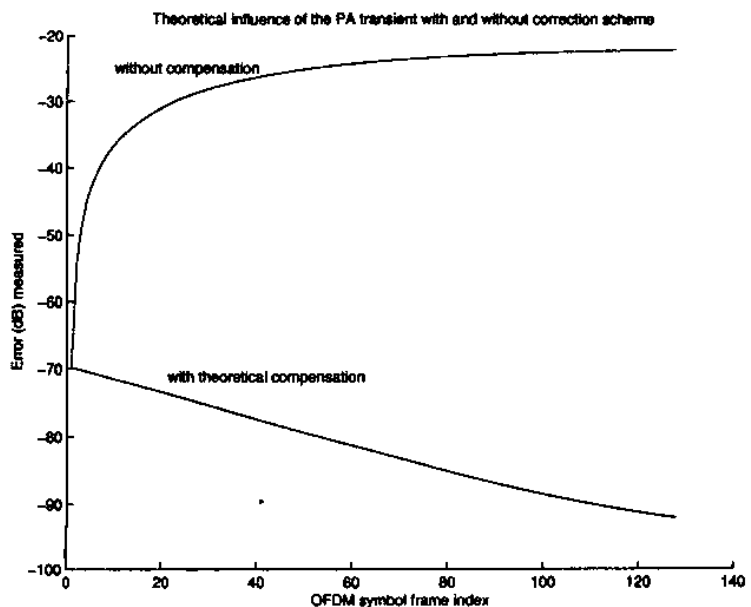

Fig. 3. Theoretical influence of the power-amplifier transient with and without correction.

\section{CORRECTION SCHEME FOR POWER-AMPLIFIER TRANSIENT}

The analysis of the effects of the power-amplifier transient above shows that it does adversely affect the system performance. We propose a correction scheme in the receiver, based on the fact that power-amplifier transient causes little ICI. However, before investigating such a scheme it is important to show that the transmission system remains compliant to the HL/2 standard, even, in the presence of the power-amplifier transient.

The basic parameter in the HL/2 standard that must be respected is the modulation accuracy, which is equivalent to EVM. We implemented a HL/2 simulator including the effects of the RF components (such as noise and non-linear effects) on the performance, which we used to estimate the performance degradation due to the power-amplifier transient with a realistic model of the RF components. A system that conforms to the HL/2 standard must have a transmitter EVM that is better than $24 \mathrm{~dB}$ [3]. The exact method to measure the conformance is not defined yet [4], and for this reason we chose to measure the conformance to the EVM specification in a manner similar to the 802.11a specification [5]. Thus the EVM is averaged over at least $20 \mathrm{HL} / 2$ frames that contain 16 symbols. As 16 OFDM symbols is relatively short in comparison to the transient seen in Fig 1, we also present the EVM for frames containing 128 OFDM symbols. The EVM as measured by our simulator can then be seen in Table 1 .

TABLE 1: EVM with and without power-amplifier transient

\begin{tabular}{c|c|c} 
OFDM Symbols & No Transient & With Transient \\
\hline 16 & $30.5 \mathrm{~dB}$ & $29.1 \mathrm{~dB}$ \\
128 & $30.0 \mathrm{~dB}$ & $24.2 \mathrm{~dB}$
\end{tabular}

It can be seen that the power-amplifier transient has little effect on the EVM when the HL/2 frame contains only 16 symbols. The degradation due to the transient is however clearly visible with 128 OFDM symbols. In both cases, the EVM specification of $\mathrm{HL} / 2$ is respected, and so the use of a correction scheme for the power-amplifier transient in the receiver can be justified.

The correction scheme involves measuring digitally the power level of the pilot carriers of the OFDM signal. This average power level is then used to normalize the constellation decision levels on an OFDM symbol by symbol basis. The advantage of this scheme is that the power level measurement can reuse existing micro-code that is used for OFDM phase tracking, and the correction itself involves only a single vector multiplication. Thus the cost to implement this scheme is minimal. The correction scheme is formally described in the following equations:

$$
\begin{gathered}
\hat{\mathbf{H}}(0)=\mathbf{R}(0) \otimes \mathbf{S}^{*}(0) \\
\alpha(k)=\sqrt{\frac{\sum_{i \in P_{i l}} R_{i}(k) \hat{H}_{i}^{*}(0) S_{i}^{*}(k)}{\sum_{i \in \text { Pil }}\left|\hat{H}_{i}(0)\right|^{2}}} \\
\mathbf{Z}(k)=\alpha(k) \hat{\mathbf{H}}^{*}(0) \otimes \mathbf{R}(k)
\end{gathered}
$$

where $\otimes$ denotes the Schur product of 2 vectors (component wise product) and * the conjugate operator. The first equation represents the simplest means to obtain an initial channel estimation, performed on symbol 0 of the frame. All these operations are illustrated Fig 2.

In the correction scheme it is important to note that it basically amounts to changing the equalization taps in the frequency domain, that are normally fixed, to a vector that 
needs to be weighted by the variable gain $\alpha(k)$, updated as each new OFDM block is received. Of course, the Viterbi metric used in the decoder needs to be weighted accordingly. Thus this scheme is preferred to a simple gain multiplication by $1 / \alpha(k)$ of all the carriers in the receiver. This correction is similar to a previously proposed pilot tracking scheme [6] for channel tracking. However, additional OFDM pilots are not needed for the case of power-amplifier transient tracking and so this correction scheme can be applied to the HL/2 and 802.11a standards as they are.

Fig 3 underlines the theoretical efficiency of the proposed power-amplifier transient canceling scheme in the noiseless case under perfect synchronization assumption and justifies its use in the practical case. It can be noticed that power-amplifier transient rejection is almost complete. It should be noted that this scheme assumes a perfect synchronization and so only the gain of the transient has been taken into account since normally the phase component should be cancelled by the phase tracking algorithms of the synchronization algorithms.

We have implemented this scheme in our $\mathrm{HL} / 2$ simulator and run simulations with realistic RF models. As the channel model plays an important part in the overall effect of the power-amplifier transient, the simulations were run using a BRAN-A [3], channel model. Propagation losses were treated with the model proposed by the ITU [7]. Fig 4 then shows the performance in terms of the error rates for various transmit powers. It can be clearly seen that the power-amplifier adversely affects the system performance. It can also be seen that the proposed correction completely recovers the lost performance.

\section{CONCLUSIONS}

We have identified the adverse effect of the poweramplifier transient, due to the switching on and off of the amplifier during operation to conserve power. It has been shown that a noise floor of roughiy $23 \mathrm{~dB}$ is introduced into the system with a commercially available amplifier, driven to simulate a typical mobile application. This level of error is sufficient to adversely affect the performance of QAM64 modulation in IEEE 802.1 la and HIPERLAN/2. However, the effect of the transient on the transmitter EVM is acceptable under the existing specifications

A receiver based correction scheme for this effect has been proposed and shown to be effective in completely correcting the effect of the power-amplifier transient. This scheme is simple to implement and reuses much of the existing phase tracking micro-code in existing $5 \mathrm{GHz}$ systems.

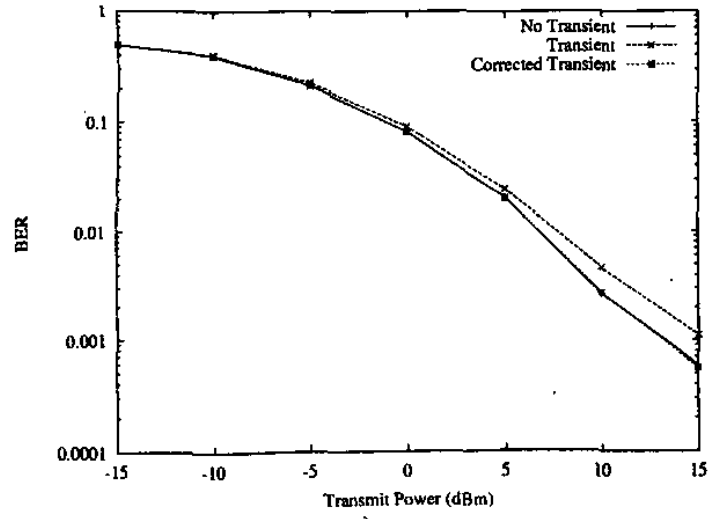

a)

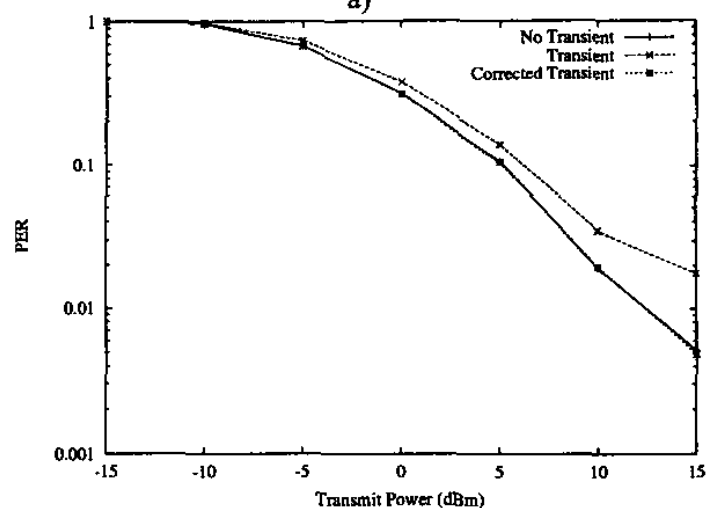

b)

Fig. 4 a) bit error rate (BER), b) packet error rate (PER) of a QAM64 signal on a BRAN-A channel [3], with an ITU-P1238 [7] propagation model and $5 \mathrm{~m}$ between receiver and transmitter.

\section{REFERENCES}

[1] M Alard and R Lassale, "Principes of modulation and channel coding for digital broadcasting for mobile receivers", European Broadcasting Union Review, vol 224, Aug, 1987, p168-190

[2] $\mathrm{Z}$ Wang and G.B Giannakis, "Wireless multicarrier communications: When Fourier meets Shannon", IEEE Sig Proc Mag, Vol 17, No 3, May 2000

[3] ETSI BRAN; "HIPERLAN Type 2, Physical (PHY) Layer", ETSI TS 101 475, April 2000.

[4] ETSI BRAN, "Part 2: Harmonized EN covering essential requirements of article 3.2 of the R\&TTE Directive" ETSI EN 020002-2 V0.f, October 2000.

[5] IEEE Std 802.11a-1999, "Part 11: Wireless LAN medium access control (MAC) and physical layer (PHY) specifications", IEEE New York, January 1999.

[6] M Sandell and O Edfors, "A comparitive study of pilot-based channel estimators for wireless OFDM", Research Report TULEA 1996, Lulea Univ of Tech

[7] ITU-R, "Propagation data and prediction models for the planning of indoor radiocommunications systems and radio local area networks in the frequency range $900 \mathrm{MHz}$ to 100GHz", ITU:R, 1997, ITU-R P.1238 